\title{
DUX4c, an FSHD candidate gene, interferes with myogenic regulators and abolishes myoblast differentiation
}

\author{
Darko Bosnakovski ${ }^{a}$, Sarah Lamb ${ }^{\text {a }}$, Tugba Simsek ${ }^{a}$, Zhaohui Xu ${ }^{\text {a }}$, Alexandra Belayew ${ }^{\text {b }}$, \\ Rita Perlingeiro ${ }^{a}$, Michael Kyba ${ }^{a}, *$ \\ a Department of Developmental Biology, UT Southwestern Medical Center Dallas, 5323 Harry Hines Blvd., TX 75390-9133, USA \\ ${ }^{\mathrm{b}}$ Lab. Biologie Moleculaire, Universite de Mons-Hainaut Pentagone, 7000 - Mons, Belgium
}

\section{A R T I C L E I N F O}

\section{Article history:}

Received 28 April 2008

Revised 17 June 2008

Accepted 18 July 2008

Available online 6 August 2008

\section{Keywords:}

Facioscapulohumeral muscular dystrophy DUX4c

Myoblast

\begin{abstract}
A B S T R A C T
Facioscapulohumeral muscular dystrophy (FSHD) is an autosomal dominant neuromuscular disease. It maps to the D4Z4 repeat array at $4 \mathrm{q} 35$, and correlates with a repeat contraction which derepresses transcription of local genes. Which, if any, of these genes is pathogenic to muscle, and through what molecular mechanism is unknown. The present study investigates the function of one candidate gene, DUX4c, encoded by a truncated inverted D4Z4 element located $42 \mathrm{~kb}$ proximal to the D4Z4 repeats. Using a gain of function approach we tested DUX4c for toxicity and effects on differentiation in C2C12 myoblasts. DUX4c-expressing myoblasts appear morphologically normal but have reduced expression of myogenic regulators, including MyoD and Myf5. Consistent with this, DUX4c-expressing myoblasts are unable to differentiate into myotubes. Furthermore, overexpression of Myf5 or MyoD rescued myoblast differentiation, suggesting that reductions in expression of these regulators are the relevant DUX4cinduced physiological changes that inhibit differentiation. Our results suggest that upregulation of DUX4c can have a deleterious effect on muscle homeostasis and regeneration, and point to a possible role for DUX4c in the pathology of FSHD.
\end{abstract}

(C) 2008 Elsevier Inc. All rights reserved.

\section{Introduction}

Facioscapulohumeral muscular dystrophy (FSHD) is a dominant inherited neuromuscular disease with a prevalence of 1 in 20,000, which makes it the third most common myopathy. It is characterized by uneven and progressive weakness and atrophy of facial, shoulder and upper arm muscle as well as in some cases with retinovasculopathy, mental retardation and epilepsy. The underlying molecular mechanism of the disease is still unknown but $95 \%$ of patients carry a subtelomeric deletion on chromosome 4q35 (Wijmenga et al., 1992). This region is mainly composed of a tandem repeat array consisting of $3.3 \mathrm{~kb}$ units (D4Z4). Each D4Z4 unit is GC rich and contains a repetitive element related to the heterochromatic LSau sequence and binding sites for a transcriptional repressor complex containing YY1, HMGB2, and nucleolin (Gabellini et al., 2002). In the unaffected population, the D4Z4 repeat array is polymorphic and may contain between 11 and over $100 \mathrm{U}$. Due to internal deletions, FSHD patients carry only 1 to 10 U (Hewitt et al., 1994).

One of the prevailing models for the mechanism of FSHD is that the normally large D4Z4 array nucleates a repressive chromatin and contractions of this array disable this repression, resulting in upregulation or derepression of local genes. Consistent with this

\footnotetext{
* Corresponding author. Fax: +1 6126248118.

E-mail address: kyba@umn.edu (M. Kyba).
}

model, Gabellini et al. observed that 2 nearby genes (FRG1 and FRG2) and one gene from $5 \mathrm{Mb}$ upstream (ANT1) were upregulated in FSHD muscle (Gabellini et al., 2002). Furthermore, over expression of FRG1 from the human skeletal actin promoter was later shown to be deleterious, leading to muscular dystrophy phenotype in transgenic mice (Gabellini et al., 2006). However, the relevance of this transgenic model, which has very high-level expression of FRG1 to FSHD pathology, has been debated as similar levels of FRG1 have not been demonstrated in FSHD muscle.

The D4Z4 repeats contain an open reading frame with two homeoboxes (DUX4) (Gabriels et al., 1999). Homeodomain proteins regulate many important developmental processes including muscle development (Buckingham et al., 2003). Remarkably the homeodomains of DUX4 have a high degree of similarity with Pax3 and Pax7, the critical homeodomain regulators of muscle development and regeneration. Recently, expression of DUX4 RNA and protein have been selectively detected in primary myoblasts from FSHD patients suggesting its involvement in FSHD (Kowaljow et al., 2007; Dixit et al., 2007). Furthermore over expression of DUX4 in different cell lines induced apoptosis (Kowaljow et al., 2007). A truncated and inverted D4Z4 repeat is located approximately $42 \mathrm{~kb}$ centromeric from the tandem D4Z4 repeats and just proximal of FRG2 (Fig. 1A) (Wright et al., 1993). This repeat also contains an ORF, referred to as DUX4c, which encodes a protein identical to DUX4 from the N-terminus through the homeodomains, but the last 82 amino acids have been 
substituted for an unrelated 32 amino acid sequence. Preliminary data suggests that DUX4c may be expressed in FSHD samples (Ansseau et al., 2006).
In this study, using a conditional, regulated gene expression system, we have analyzed the effect of DUX4c expression on $\mathrm{C} 2 \mathrm{C} 12$ myoblast differentiation. We demonstrate that DUX4c down-

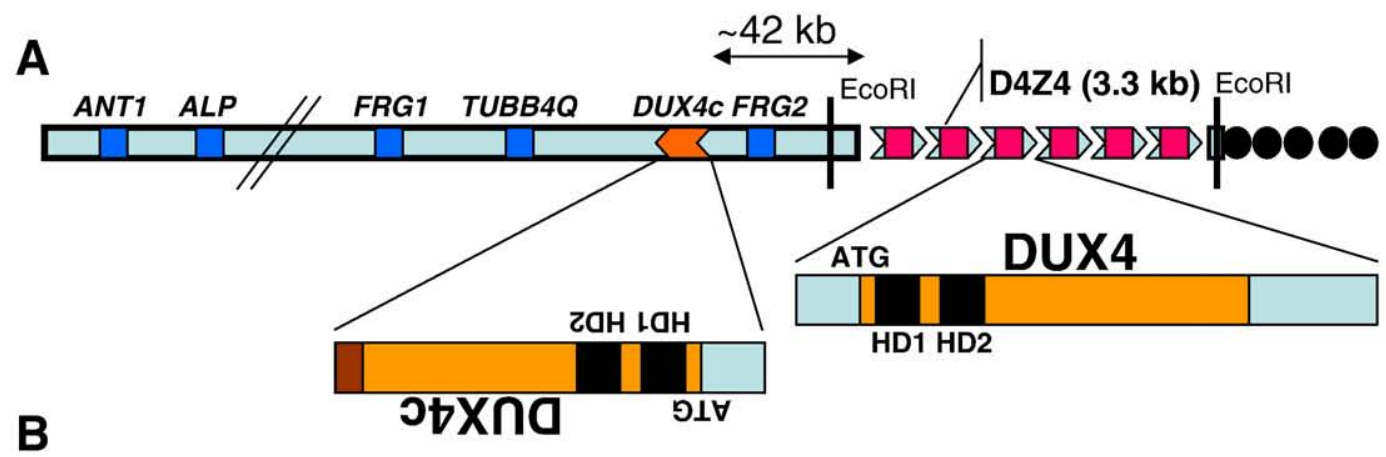

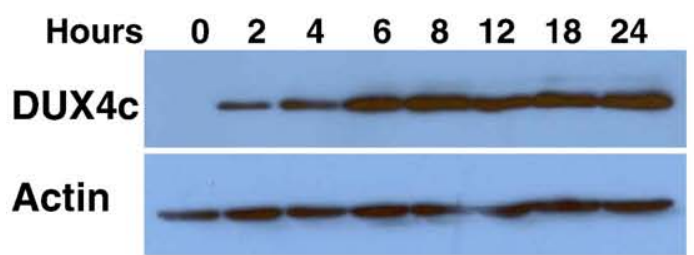

C
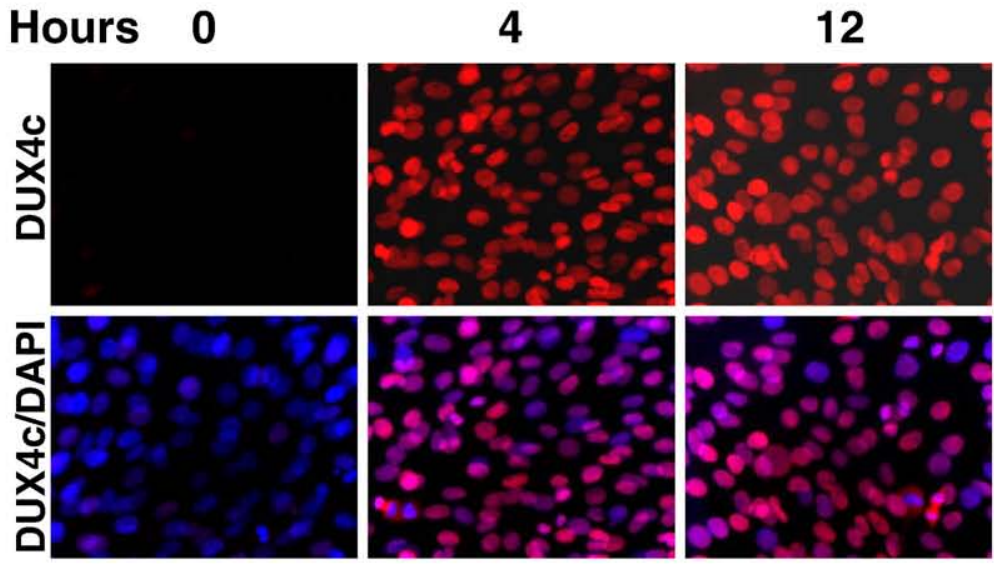

E

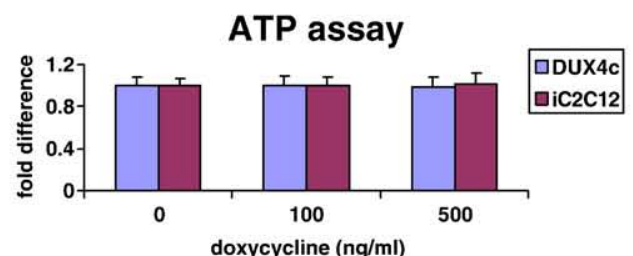

\section{Cell count (iC2C12-DUX4c)}

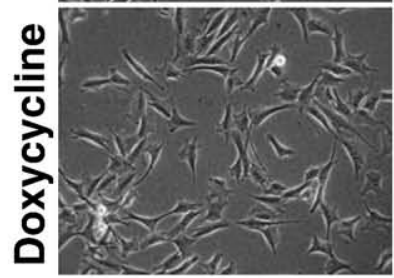

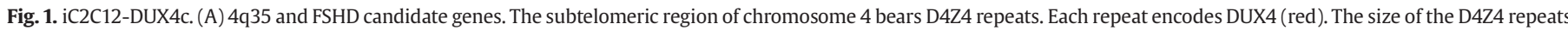

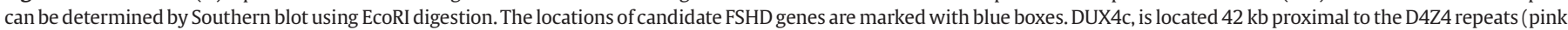

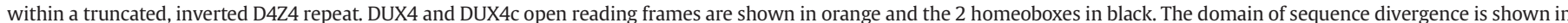

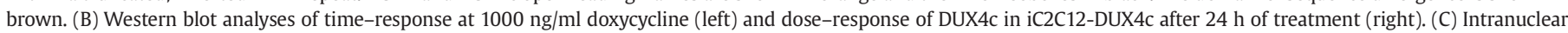

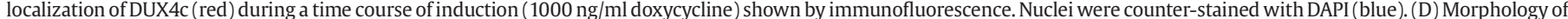

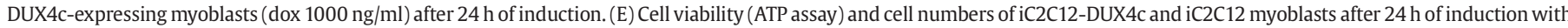

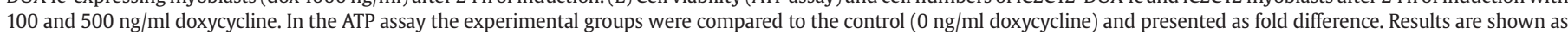
average \pm STDEV (ATP assay, $n=8$; cell counts, $n=3$ ) 
A

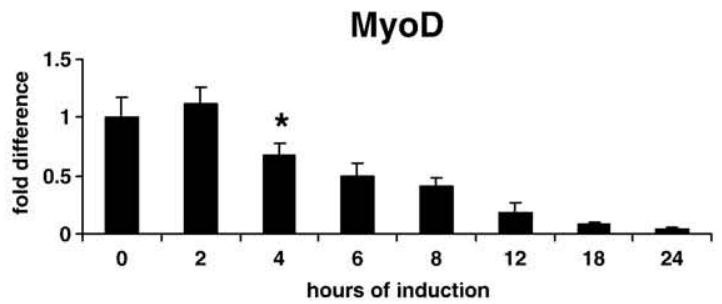

B

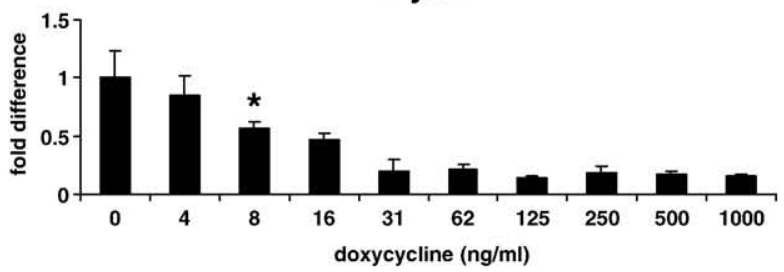

C

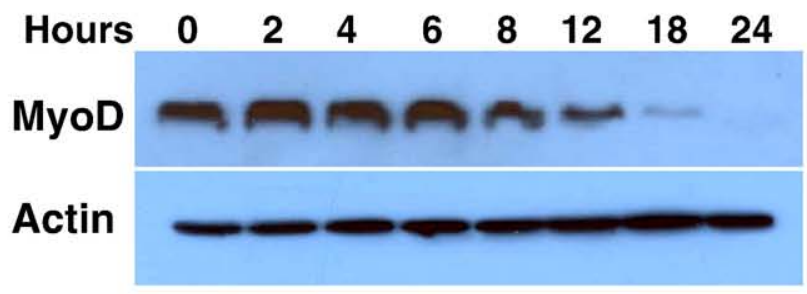

Myf5

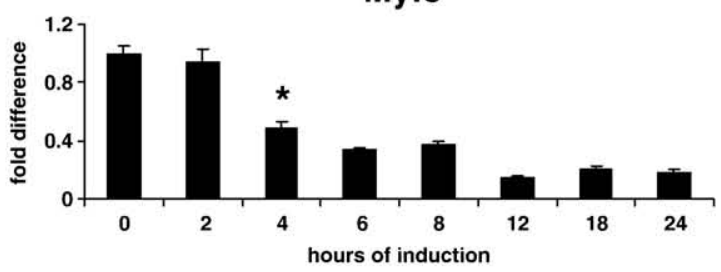

Myf5

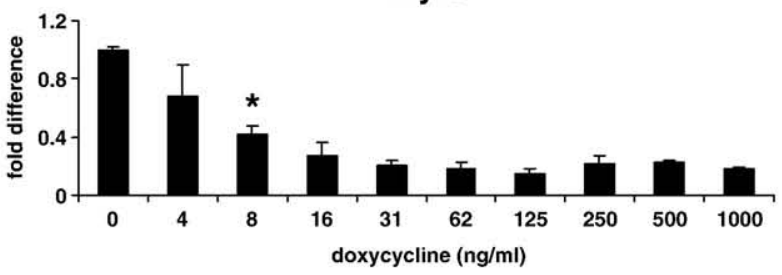

ng/ml $\quad 0 \quad 4 \quad 8 \quad 8 \quad 16 \quad 3264125250500$

MyoD

Actin

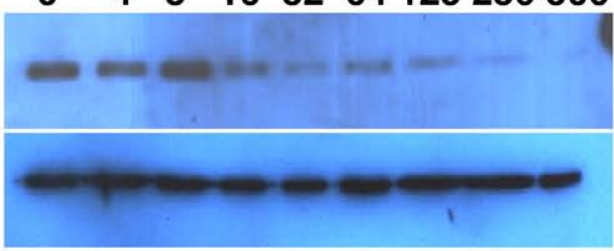

12
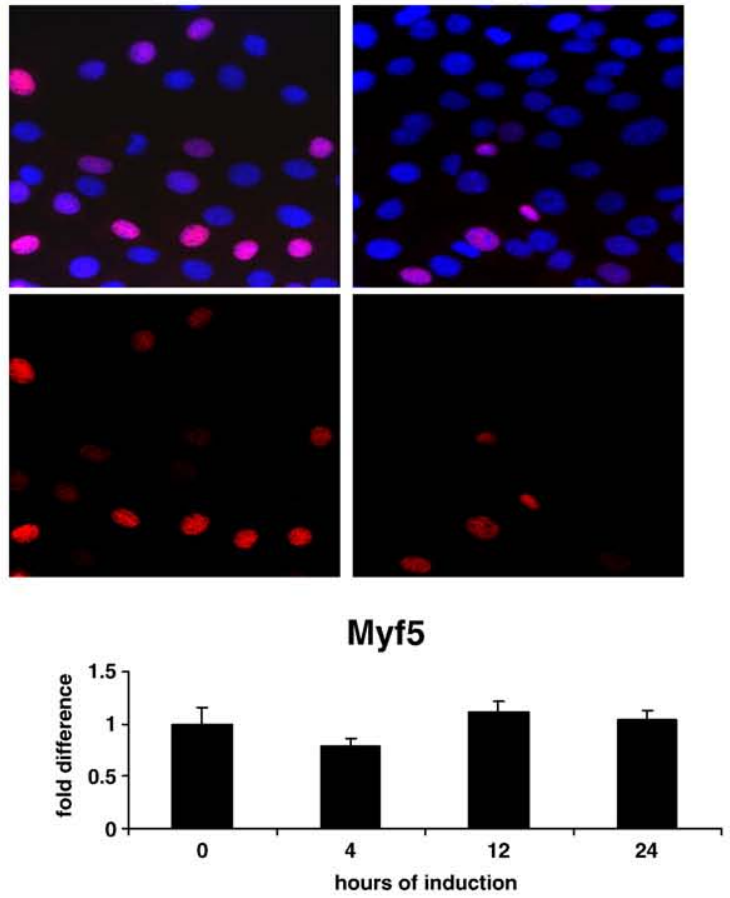

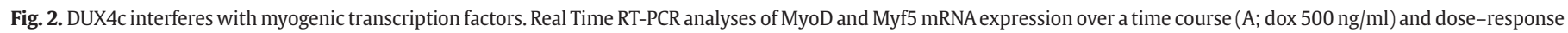

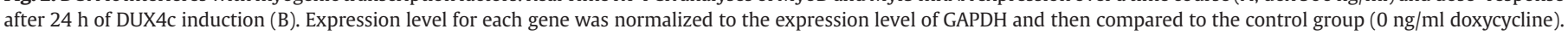

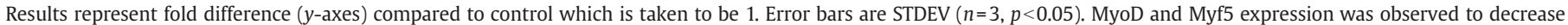

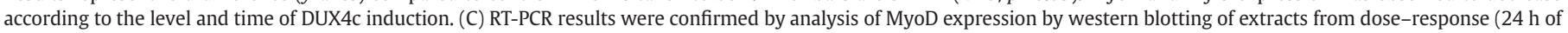

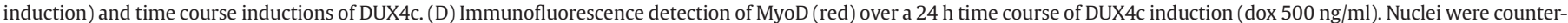

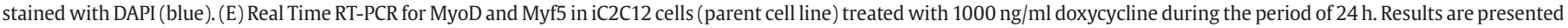
as fold difference compared to the control $(0 \mathrm{ng} / \mathrm{ml})$.

regulates expression of myogenic transcriptional factors MyoD, Myf5 and myogenin. This interaction with myogenic regulators correlates with diminished myoblast fusion and terminal myotube differentia- tion even at low levels of DUX4c expression. Overexpression of MyoD or Myf5 in DUX4c-expressing cells rescued their ability to differentiate suggesting that they are the key downstream genes that mediate this 
effect. Our results predict that DUX4c expression would have a deleterious effect on muscle regeneration, and could therefore play a role in FSHD pathogenesis.

\section{Results and discussion}

\section{DUX4c-inducible myoblasts}

We modified mouse C2C12 myoblasts to enable conditional DUX4c expression by inserting the cDNA for DUX4c into the inducible locus of iC2C12, a $\mathrm{C} 2 \mathrm{C} 12$ subclone that expresses rtTA and carries a tetracycline-inducible locus that can be targeted by cre-lox recombination (manuscript submitted). The parent iC2C12 cell line has a cassette exchange recombination locus which allows efficient gene targeting. The integration site of this locus was selected for absence of leakiness and minimal silencing. The principal advantage of this system is that genes can reliably and predictably be expressed in a conditional dose-dependent manner. Because DUX4c, like DUX4, is likely to be expressed at very low levels (Kowaljow et al., 2007; Dixit et al., 2007), it is critical to be able to titrate expression down to levels barely detectable by western blot. However, to facilitate the evaluation of downstream target genes and other potential physiological effects, rapid high-level expression is also necessary. We evaluated expression in the DUX4c-inducible myoblasts, which we named iC2C12-DUX4c, using a monoclonal antibody that was raised against DUX4 and cross reacts to DUX4c (Dixit et al., 2007). When exposed to doxycycline, iC2C12-DUX4c cells overexpress human DUX4c in a dose-dependent manner with rapid kinetics (Fig. 1B). At high levels of expression (1000 ng/ml doxycycline) DUX4c was expressed in virtually all cells within $4 \mathrm{~h}$, and was exclusively localized to the nucleus, as shown by immunofluorescence (Fig. 1C). Homeodomain proteins are nuclear transcription factors, and in many cases localization depends on a nuclear localization signal (NLS) residing within the homeodomain itself (Hessabi et al., 1999). In the case of DUX4, fluorescence photobleaching of a constitutively expressed GFP fusion protein showed that the DUX4 homeodomain contains an NLS, and that the protein resides primarily in the nucleus (Ostlund et al., 2005).

At $24 \mathrm{~h}$ post-induction, the morphology of DUX4c-expressing myoblasts appeared normal (Fig. 1D) and cell viability as measured by an ATP assay was unaffected (Fig. 1E). To control for doxycyclinespecific effects that might be unrelated to DUX4c expression, we tested the iC2C12 parent (non-DUX4c-expressing) cell line, and no doxycycline-induced changes were observed (Fig. 1E). Furthermore, after 6 days of continual exposure to doxycycline, we did not detect any changes in morphology or viability of the parent iC2C12 cells (data not shown). Unlike DUX4c and DUX1, DUX4 is toxic in myoblasts and its expression correlates with apoptosis, indicated by activation of caspases 3 and 7 followed by increased lactate dehydrogenase (LDH) activity and positive staining for Annexin V (Kowaljow et al., 2007 and D.B., M.K., unpublished results). FHSD-affected myoblasts have been reported to exhibit morphological abnormalities, including a swollen and irregular cytoplasm containing numerous vacuoles (Winokur et al., 2003a). Given the lack of morphological changes in iC2C12DUX4c cells even at high levels of DUX4c expression, the abnormal morphology of FSHD-affected myoblasts is therefore unlikely to be due to DUX4c, and is probably mediated by other candidate genes, including DUX4.

\section{DUX4c interferes with myogenic transcriptional factors}

We next evaluated whether DUX4c expression had any effect on the ability of $\mathrm{C} 2 \mathrm{C} 12$ myoblasts to differentiate into multinucleated myotubes. This process involves the sequential activation of myogenic regulatory factors by the homeodomain-containing transcription factors Pax3 and Pax7, therefore we first evaluated expression of MyoD and Myf5, two well known targets of Pax3 and Pax7 (Tajbakhsh et al., 1997; Bajard et al., 2006). In proliferating myoblasts, the levels of expression of MyoD and Myf5 were inhibited by DUX4c, starting as early as $4 \mathrm{~h}$ post-induction with $500 \mathrm{ng} / \mathrm{ml}$ doxycycline (Fig. 2A). This inhibition increased in a time- and dose-dependent manner (Figs. 2A and 2B). Significant down-regulation of Myf5 and MyoD was detected in the cells treated with as little as $8 \mathrm{ng} / \mathrm{ml}$ doxycycline for $24 \mathrm{~h}$ (Fig. 2B). By western blot and immunofluorescence, down-regulation of MyoD protein was evident within $8 \mathrm{~h}$ of high-level $(500 \mathrm{ng} / \mathrm{ml}$ doxycycline) DUX4c induction (Figs. 2C and 2D). Even lower doses of DUX4c (16-32 ng/ml of doxycycline) were sufficient for almost complete suppression of MyoD within 24 h (Figs. 2BB and 2C). On the other hand, doxycycline by itself did not have any significant influence on MyoD or Myf5 in the non-DUX4c-expressing parent cell line, iC2C12 (Fig. 2E).

Expression profiling in FSHD patients has demonstrated that genes with altered expression include many involved in myogenic differentiation and represent targets of the muscle-specific transcriptional factor MyoD (Winokur et al., 2003b). Different subsets of MyoD target genes were affected in different groups of FSHD patients, categorized according to the number of the remaining D4Z4 repeats (Celegato et al., 2006). For this reason it has been proposed that interference with an early sub-program of MyoD-mediated muscle cell differentiation is one mechanism underlying FSHD (Winokur et al., 2003b). Our results show a strong correlation between DUX4c and MyoD, with DUX4c expression inducing rapid down-regulation of MyoD (Fig. 2). MyoD and Myf5 are skeletal muscle-specific genes which belong to the basic-helix-loop-helix family of proteins and are necessary and sufficient for myogenesis (Weintraub et al., 1991). Mice lacking both MyoD and Myf5 have a complete absence of skeletal muscle, demonstrating a critical role for these factors in myogenesis (Rudnicki et al., 1993). Taken together, the importance of MyoD in myogenesis and its misregulation in FSHD makes this property of DUX4c particularly interesting and possibly a factor in the pathology of FSHD. The reduced expression of MyoD may be explained by a direct interaction between DUX4c and the MyoD gene, although we have no evidence for this at the present time, or it may be mediated by an intermediary factor.

\section{DUX4c abolishes myogenic differentiation}

The ability of DUX4c to inhibit MyoD and Myf5 expression suggested that DUX4c-expressing myoblasts may show a phenotype

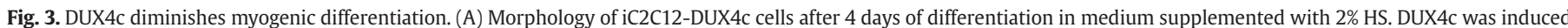

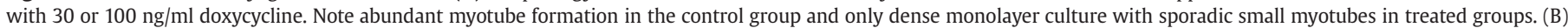

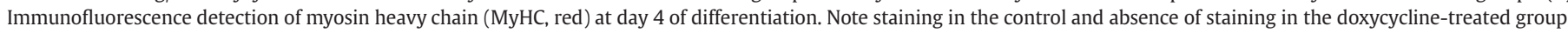

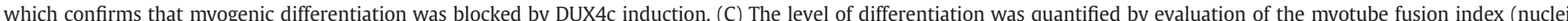

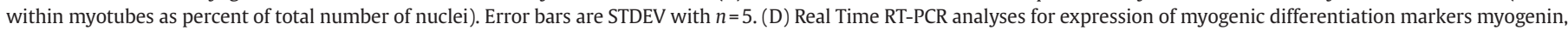

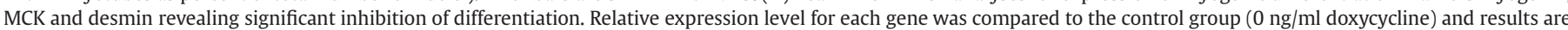

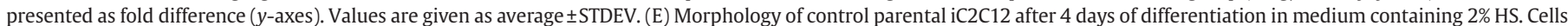

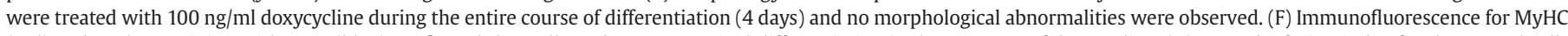

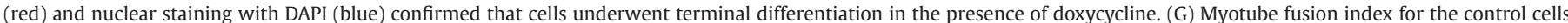

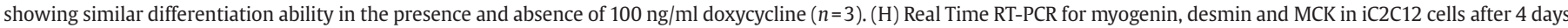

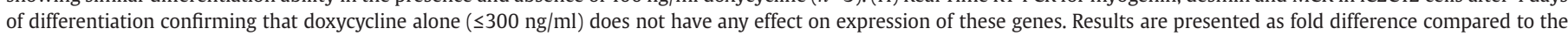
control (0 $\mathrm{ng} / \mathrm{ml}$ doxycycline). 
when induced to differentiate. We tested the ability of DUX4cexpressing myoblasts to differentiate into myotubes by growing them to confluence and withdrawing growth factors (switching to medium supplemented only with $2 \%$ horse serum). In the absence of doxycycline, iC2C12-DUX4c cells differentiated en masse into multinucleated myotubes, however in the presence of doxycycline, added at the time differentiation was induced, cells failed to fuse and did not form myotubes (Fig. 3A). The inhibitory effect was morphologically

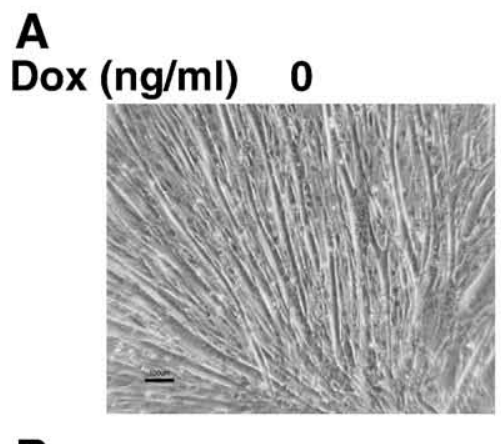

B

Dox (ng/ml) 0
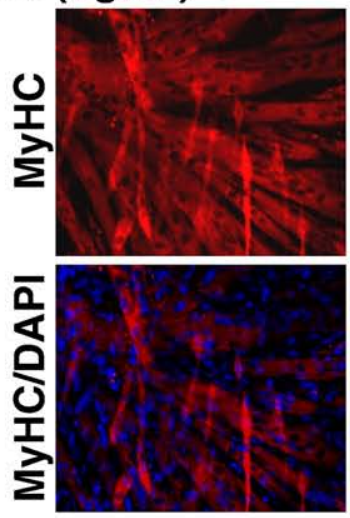

D

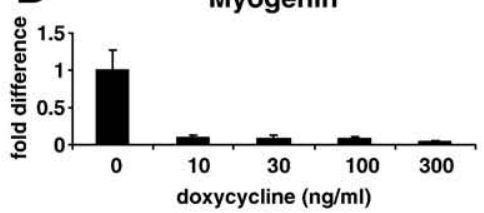

E

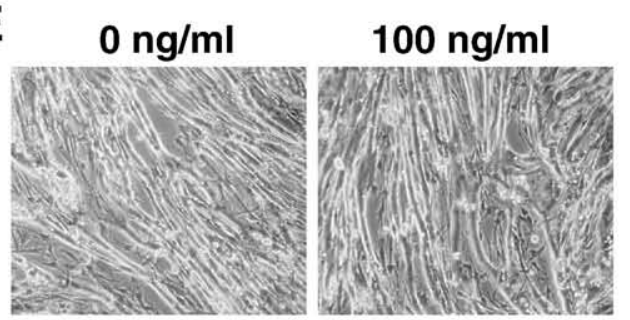

G
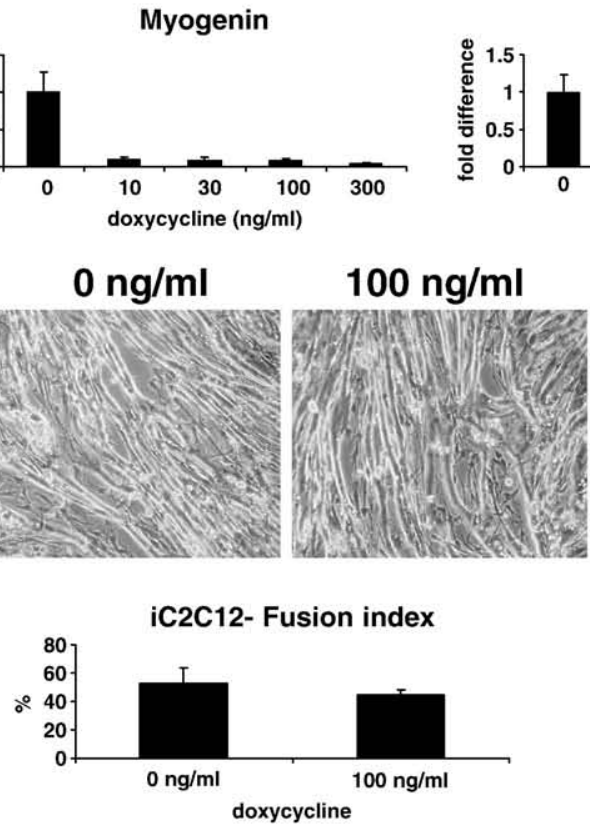

30

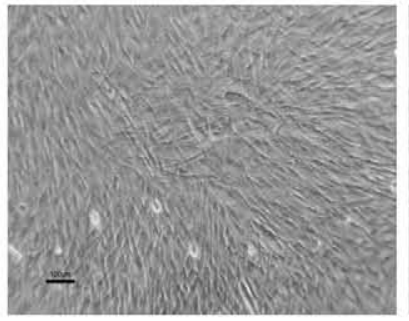

30

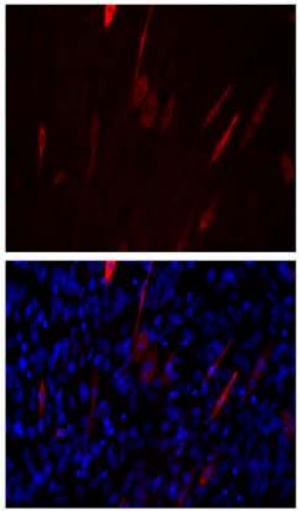

Desmin
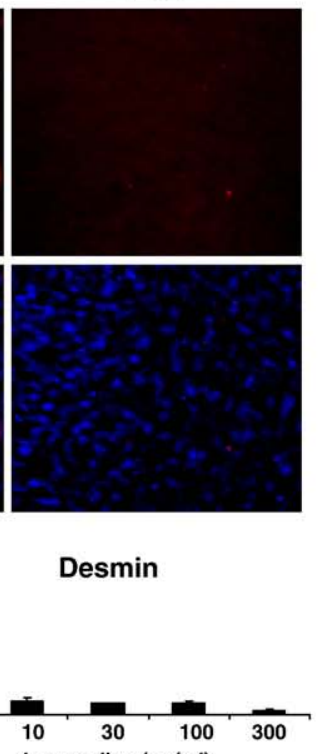
doxycycline $(\mathrm{ng} / \mathrm{ml})$

100
100

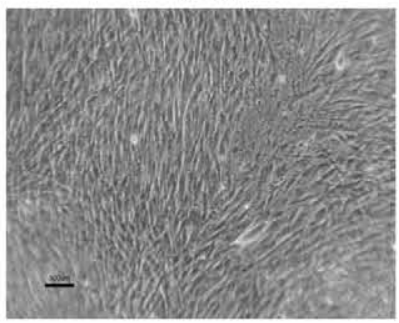

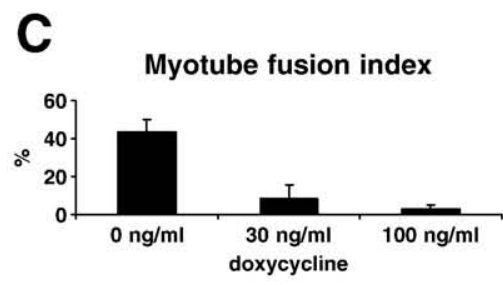

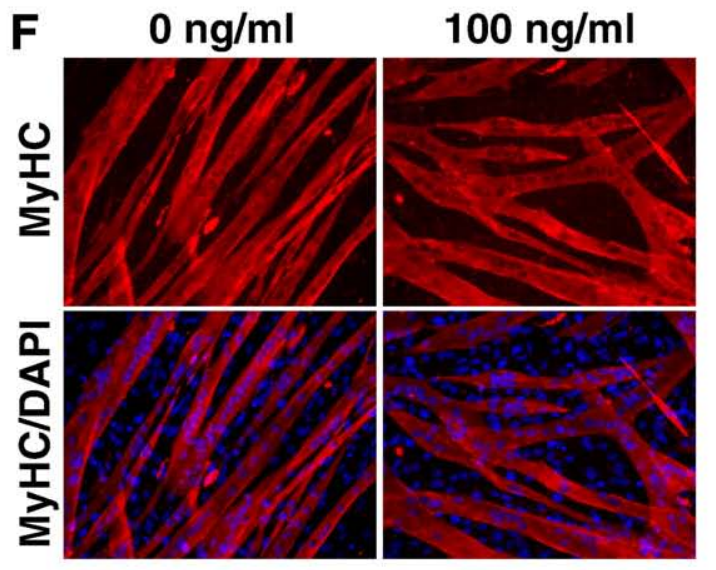

Desmin

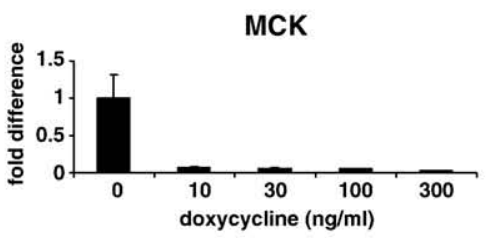

H Myogenin
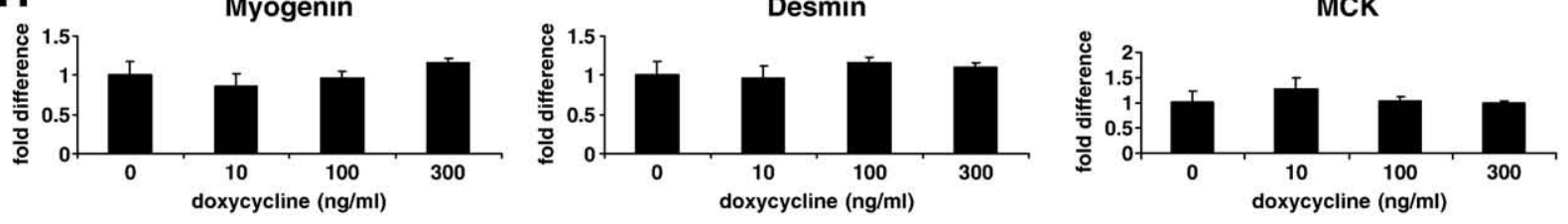
evident over the entire process of differentiation (4-6 days). Remarkably, the effect was significant even when the cells expressed low levels of DUX4c (30 ng/ml doxycycline) in which only sporadically fused small myotubes were observed (Fig. 3A). The effect on differentiation was dose-dependent and more severe with higher DUX4c expression.

To determine whether differentiation per se was blocked, or cells were merely failing to fuse, we evaluated molecular markers of differentiation. The differentiation defect was supported by immunofluorescence for myosin heavy chain (MyHC), which was either sporadic (30 ng/ml dox) or completely absent (100 ng/ml, Fig. 3B). It was also supported by a greatly reduced myotube fusion index (percent of nuclei within multinuclear myotubes, Fig. 3C). Untreated iC2C12-DUX4c, the control group, differentiated at the expected level with $50-60 \%$ of nuclei present in fusion derivatives (Fig. 3C and Fig. 4G). In addition, Real Time RT-PCR revealed significantly reduced expression of myogenin, desmin and muscle creatine kinase (MCK) even in samples which were treated with only $10 \mathrm{ng} / \mathrm{ml}$ doxycycline (Fig. 3D). At this low concentration of doxycycline, DUX4c is barely detectable by western blot. This is significant, as the relevant FSHD candidate gene may be expressed at very low levels and difficult to detect in patient biopsies. To control for potential effects of
A

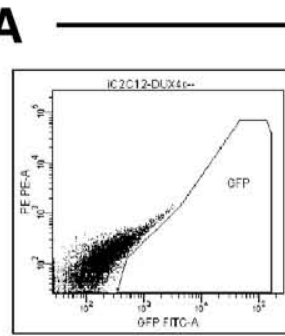

iC2C12-DUX4c

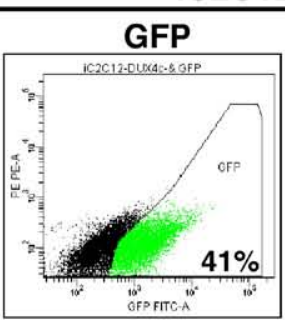

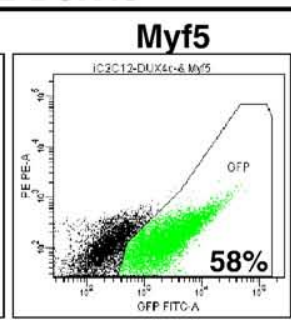
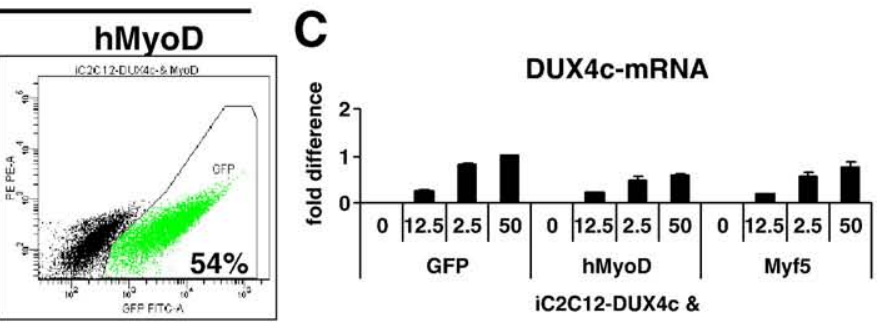

B

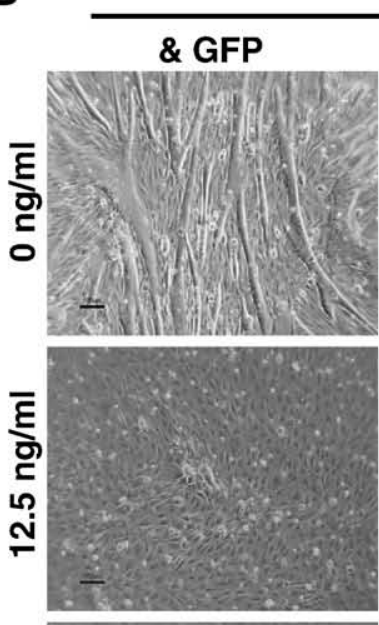

iC2C12-DUX4c
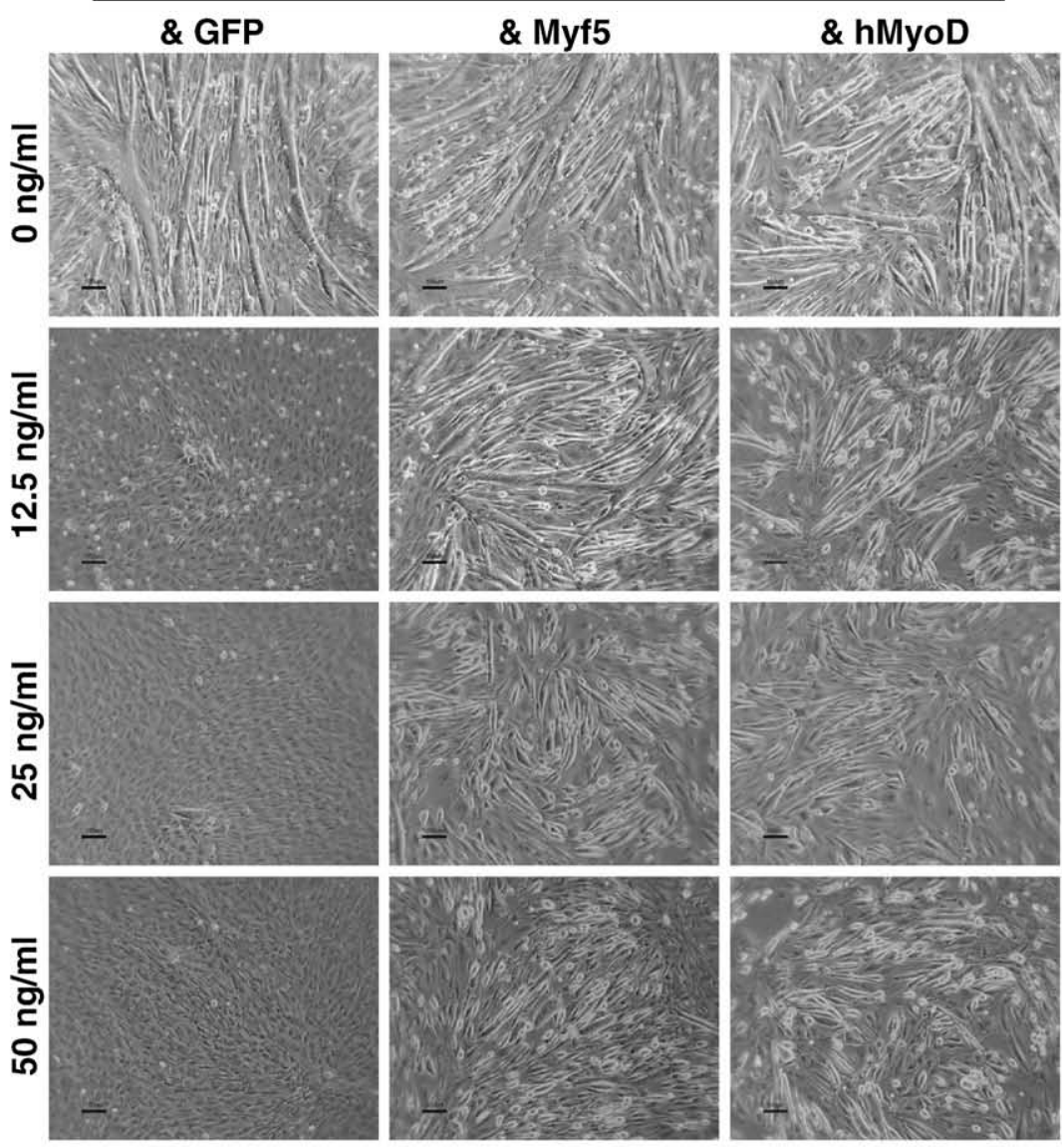
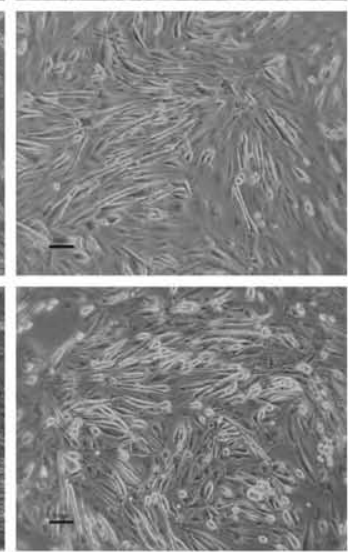

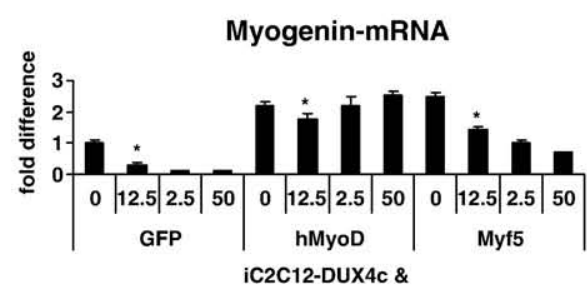

MCK-mRNA
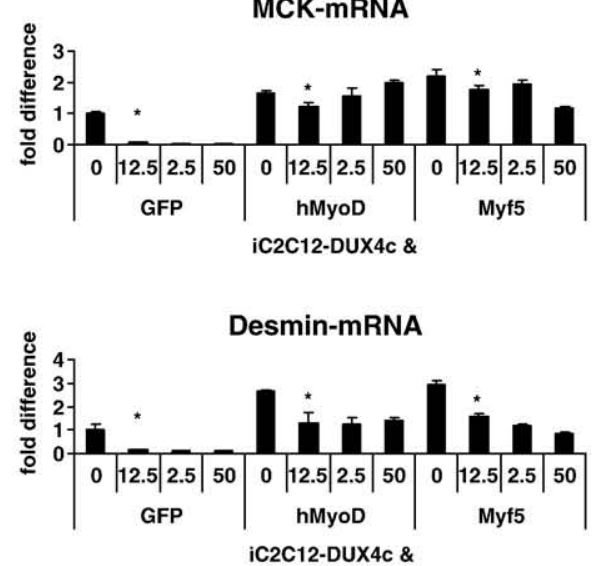

mMyoD-mRNA

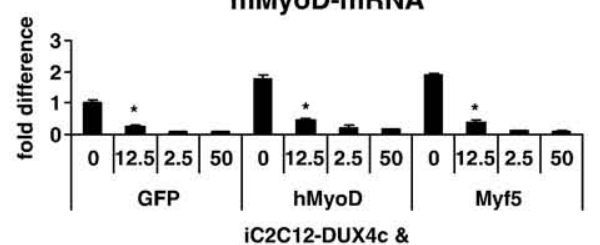

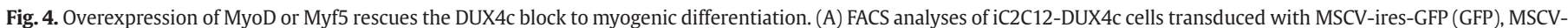

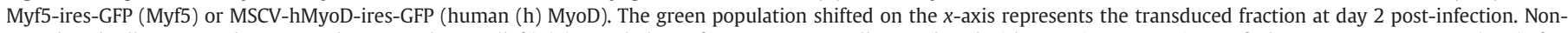

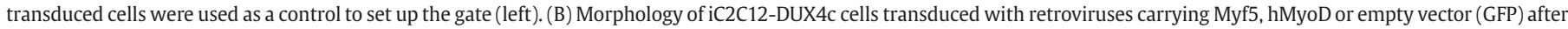

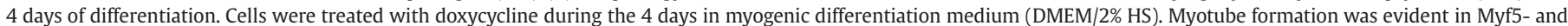

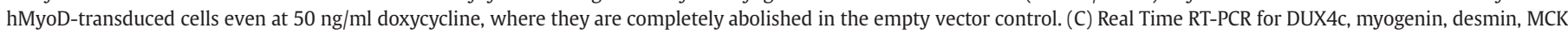

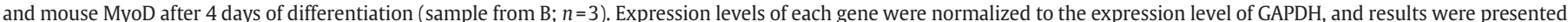

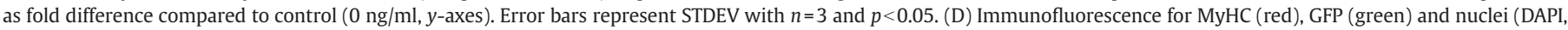

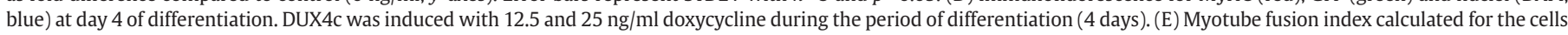
shown in $4 \mathrm{D}$. 

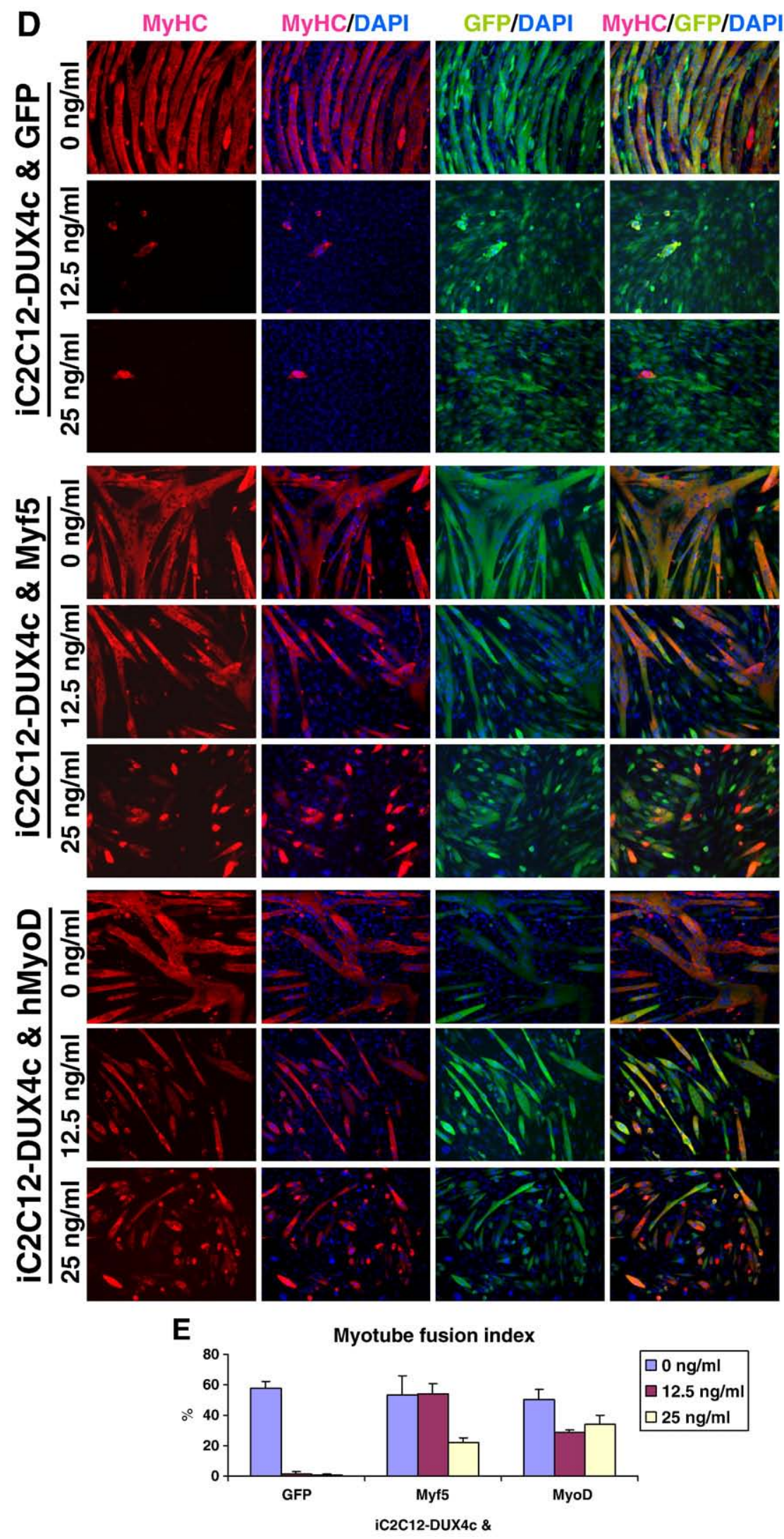

Fig. 4 (continued).

doxycycline on myoblast differentiation we performed the same sets of experiments with the parental non-DUX4c-expressing iC2C12 cell line. During 4 days of differentiation, the presence of $100 \mathrm{ng} / \mathrm{ml}$ doxycycline had no effect (Fig. 3E). Positive immunofluorescence staining for MyHC confirmed that doxycycline-treated iC2C12 (parental control) cells differentiated appropriately into multinuclear 
myotubes (Fig. 3F). We did not detect any significant alteration in the myoblast fusion index in response to doxycycline alone (Fig. 3G) nor in the expression of myogenic differentiation markers by Real Time RTPCR analyses (Fig. 3H). We therefore conclude that these effects are specific to DUX4c.

\section{Overexpression of MyoD or Myf5 rescues the DUX4c myogenic} differentiation blockade

Because reduced levels of MyoD and Myf5 in myoblasts under both proliferation and differentiation conditions correlated with the inability of DUX4c-expressing cells to differentiate, and because these two genes are critical regulators of myogenic differentiation, we reasoned that the principal reason for the lack of differentiation was the lack of expression of the necessary bHLH factor. To test this hypothesis, we used an independent vector to express MyoD or Myf5 in DUX4c-expressing myoblasts, and repeated the differentiation experiments, above. An MSCV retroviral vector expressing cDNAs of human MyoD, mouse Myf5, or empty control, followed by ires-GFP for tracking positive cells, was introduced into subconfluent iC2C12-DUX4c cells. Human MyoD was selected for these experiments in order to allow independent tracking of the effect of DUX4c on expression of the mouse MyoD gene at the RNA and protein level. Two days post-infection, the transduction rate was determined by FACS analysis in one arm. We used transductions in which the rate approached $50 \%$ in order to be able to measure differentiation potential of transduced (green fluorescent) and nontransduced cells side by side. Myoblasts were then switched to differentiation medium (DMEM/2\% HS) and DUX4c was induced over a dose-series. At day 4 of differentiation in the controls ( $0 \mathrm{ng} /$ $\mathrm{ml}$ doxycycline) of all of the experimental groups GFP, Myf5 or hMyoD, complete terminal myotube differentiation was observed (Fig. 4B). As expected, DUX4c-expression, even under $12.5 \mathrm{ng} / \mathrm{ml}$ doxycycline, prevented myotube formation in the empty vectortransduced cells. On the other hand, obvious differentiation, characterized by fused, elongated myotubes, was observed in hMyoD- or Myf5-transduced cells (Fig. 4B).

Real Time RT-PCR analyses for DUX4c confirmed its dosedependent induction in all samples (Fig. 4C). RT-PCR analyses for myogenin, desmin, and MCK also confirmed that differentiation was blocked in DUX4c-induced cells coexpressing GFP, but allowed in Myf5 and MyoD coexpressing cells (Fig. 4E). By using human MyoD for these studies, we were able to independently analyze expression from the mouse MyoD gene. Irrespective of exogenous Myf5 or MyoD, DUX4c was still able to repress the mouse MyoD gene, indicating that the exogenous bHLH factors were not inhibiting DUX4c activity directly, but are acting in classical epistatic fashion, downstream of DUX4c.

Differentiation was confirmed by immunofluorescence for MyHC (Fig. 4D). To demonstrate that rescue was specific to cells expressing the rescue constructs, we performed double staining for GFP and MyHC. Virtually all of MyHC positive cells were GFPpositive while undifferentiated cells were GFP-negative (Fig. 4D), indicating that rescue was specific to that portion of cells that had been transduced with the bHLH factor. To quantify the level of differentiation we analyzed the myotube fusion index. Myf5 or MyoD overexpressing cells demonstrated significant levels of myotube fusion (Fig. 4E).

It was notable that although hMyoD and Myf5 were clearly able to rescue differentiation, the extent of rescue was reduced both in terms of the myotube fusion index and in the size of individual myotubes as doxycycline was titred to higher levels. This was in spite of MyoD target genes, myogenin, desmin, and MCK being fully rescued to wild-type (no dox) levels. This suggests that additional unknown factors in addition to MyoD/Myf5, and their direct target genes, are necessary for regulating the extent of differentiation and the size of myotubes, and implies that DUX4c may interfere with these factors as well.

\section{Conclusion}

In summary, we have shown that DUX4c does not have toxic effects on myoblasts while proliferating, but that it interferes with the expression of myogenic regulators and inhibits differentiation of myoblasts, even at very low levels of expression. Diminished differentiation is in large part due to down-regulation of the crucial myogenic transcription factors MyoD and Myf5. As myogenesis is necessary for homeostatic maintenance of muscle, if DUX4c were expressed as satellite cells undergo cycles of regeneration, it would be predicted to reduce the efficiency of regeneration by interfering with differentiation. The fact that FSHD manifests in individuals with fully developed muscles means that DUX4c must not be expressed during development at levels that would completely block all differentiation, however by reducing the efficiency of differentiation, DUX4c would be predicted to place an extra burden on the stem cell compartment in FSHD-affected individuals leading to early depletion of regenerative potential. Scant data exists on the expression of DUX4c (or DUX4) in adults or embryos (Kowaljow et al., 2007; Dixit et al., 2007; Ansseau et al., 2006).

DUX4c is not likely to be solely responsible for FSHD - recent work has shown that FRG1, when overexpressed specifically in differentiated muscle of transgenic mice, causes a myopathic phenotype (Gabellini et al., 2006). The interference with regeneration described above could enhance the severity of a primary defect caused by FRG1 or another FSHD candidate gene. There has been recent debate over the relevance of FRG1, given the high levels of expression required to elicit its phenotype in mice. By inhibiting regeneration, DUX4c could potentially reveal a latent phenotype of low level expression of FRG1. Although the search for the proximal effector of FSHD has focused on identifying the key gene, it is also possible that the complicated FSHD phenotype represents the combinatorial action of more than one $4 q 35.2$ gene. It will therefore be interesting to look for synergy in transgenic models employing combinatorial low level expression of DUX4c and other FSHD candidate genes.

\section{Materials and methods}

\section{Cell culture}

C2C12 cells were cultured in proliferation medium consisting of high glucose Dulbecco's Modified Eagle Media (DMEM) supplemented with L-glutamine and sodium pyruvate (Gibco), penicillin and streptomycin (P/S, Gibco) and 20\% fetal bovine serum (FBS, Atlanta Biologicals) at $37{ }^{\circ} \mathrm{C}$ in $5 \% \mathrm{O}_{2} / 5 \% \mathrm{CO}_{2}$. For myotube formation, $\mathrm{C} 2 \mathrm{C} 12$ cells were cultured on gelatin-coated dishes in proliferation medium until confluence, and then washed with serum-free DMEM and differentiated with DMEM supplemented with $2 \%$ horse serum (HS, Sigma) and $10 \mu \mathrm{g} / \mathrm{ml}$ insulin (Sigma) for 4-6 days. Cell images were captured on an Olympus IX70 microscope (Olympus Optical, Tokyo, Japan) with a Nikon DXM1200 digital camera (Nikon, Tokyo, Japan) and Nikon ACT-1 software.

\section{Generation of DUX4c inducible myoblast cell line (iC2C12-DUX4c)}

DUX4c was subcloned from pCLneo-DUX4c as Xbal/EcoRI fragment into $\mathrm{pBS}$ in order to acquire Xhol and NotI cloning sites, which were then used for sub-cloning into p2lox to generate p2lox-DUX4c. iC2C12 cells were transfected with p2Lox-DUX4c, and selected in $800 \mathrm{ug} / \mathrm{ml}$ of G418.

\section{Cell viability and proliferation}

For ATP assay, iC2C12-DUX4c and iC2C12 were plated in a 96 well plate (2400 cells/well). DUX4c was induced the following day with 
100, 500 and $1000 \mathrm{ng} / \mathrm{ml}$ doxycycline. Cell viability was analyzed $24 \mathrm{~h}$ post-induction by measurement of ATP (Molecular Probes, Eugene, OR) following the manufacturer's protocol. To assay the ATP, medium was removed, cells were lysed with $50 \mathrm{ul}$ of ATPlite and luminescence was read on a POLARstar Optima Microplate Reader (BMG LABTECH, Offenburg, Germany). The absolute level of luminescence was normalized to the control ( $0 \mathrm{ng} / \mathrm{ml}$ doxycycline) and the results were represented as fold difference. For analyses of cell proliferation 10,000 iC2C12-DUX4c cells were plated in each well of 12-well plates. The following day DUX4c was induced by 0,100 and $500 \mathrm{ng} / \mathrm{ml}$ doxycycline for $24 \mathrm{~h}$. The cell number was determined by counting live cells with a hemocytometer $(n=3)$.

\section{Western blotting}

Cells were lysed for $30 \mathrm{~min}$ at $4{ }^{\circ} \mathrm{C}$ with RIPA buffer (Santa Cruz) supplemented with a protease inhibitor cocktail (Roche). Proteins were separated by electrophoresis on 10\% PAGE gels (Bio Rad). The gels were then transferred to PVDF membranes (Bio Rad), and membranes were blocked in 5\% skim milk in TBS-T (Tris-buffered saline with $0.01 \%$ Tween-20) for $1 \mathrm{~h}$ at room temperature. Primary antibodies: mouse monoclonal anti-DUX4 (1:2000, Dixit et al., 2007), mouse anti-MyoD (1: 250, BD Biosciences) were added in the same milk mixture and incubated overnight at $4{ }^{\circ} \mathrm{C}$. Anti mouse horseradish peroxidase-conjugated secondary antibody (1:3000, Santa Cruz) was incubated for $1 \mathrm{~h}$ at room temperature. Proteins were detected by applying ECL Plus (GE Healthcare) to the membrane and exposure to $\mathrm{X}$-ray film. For normalization, membrane was striped by $100 \mathrm{mM} 2-$ mercaptoethanol and 2\% SDS in $0.5 \mathrm{M}$ Tris buffer for $30 \mathrm{~min}$ and reprobed with monoclonal mouse anti-actin (1:2000, Sigma) antibody for $30 \mathrm{~min}$ at room temperature.

\section{Immunofluorescence}

Cells were cultured on gelatin-coated coverslips, fixed with $4 \%$ paraformaldehyde for 20 min, permeabilized by $0.3 \%$ Triton X-100 for $30 \mathrm{~min}$ and blocked by $10 \%$ goat serum for $1 \mathrm{~h}$ at room temperature. Primary antibodies (anti-DUX4, anti-MyoD and mouse anti-myosin heavy chain ( $1: 20$, Hybridoma bank)) were incubated in $\mathrm{PBS} / 2 \%$ goat serum at $4{ }^{\circ} \mathrm{C}$ overnight, followed by anti-mouse Cy3-conjugated secondary antibodies (1:400, Sigma) at room temperature for $45 \mathrm{~min}$. For detection of GFP, polyclonal chicken anti-GFP antibody (1:500, AbCam) as primary and Alexa Fluor 488 (1:500, Invitrogen) as secondary antibody were used. Cells were counter-stained using 4',6-diamidino-2-phenylindole (DAPI, Invitrogen) to visualize nuclei and mounted in "Immu-Mount" (Thermo Scientific, Pittsburg, USA). The images were visualized with an Olympus BX50 microscope, an Olympus U-CMAD digital camera, and MetaVue 5.0 software.

\section{Quantitative Real Time RT-PCR (qRT-PCR)}

Total RNA was extracted with Trizol (Invitrogen) and cDNA was generated using $1 \mu \mathrm{g}$ DNase-treated RNA with oligo-dT primers and TermoScript polymerase (Invitrogen). PCR was performed by using pre-made primers/probes (GAPDH Mm99999915_g1, MyoD1Mm00440387_m1, Myf5Mm00435125_m1, myogenin Mm00446194_m1, desmin Mm00802455_m1, MCK Mm00432556_m1, DUX4c Hs02386627_gh all from Applied Biosystems) with TaqMan Real Time PCR premixture and run on an Applied Biosystems 7500 real time PCR System. For detecting of human MyoD1, we designed intron-spanning primers (forward: cactccggtcccaaatgtag and reverse: ttccctgtagcaccacacac) and Power SYBR Green PCR Master Mix (Applied Biosystems) was used. PCR conditions were standard for the Applied Biosystems pre-made probesets: $95{ }^{\circ} \mathrm{C}$ for $15 \mathrm{~s} ; 60{ }^{\circ} \mathrm{C}$ for $60 \mathrm{~s} 7500$ real time PCR System. Glyceraldehyde phosphate dehydrogenase (GAPDH) was used as the internal standard.
Gene expression was normalized and analyzed using delta Ct method by 7500 System Software (Applied Biosystems). Results were presented as fold difference compared to the control $(\mathrm{ddCt})$ or fold difference compared to the GAPDH ( $1 / \operatorname{power}(2 / \mathrm{dCt}))$. All reactions were performed at least in triplicate and the error bar is STDEV.

\section{Cloning of Myf5 and hMyoD retroviral vectors and virus production}

Mouse Myf5 cDNA from pCR4-TOPO-Myf5 (ID 40130421, Open Biosystems) was subcloned as an EcoRI fragment into pMSCV-ires-GFP to generate pMSCV-Myf5-ires-GFP. Human MyoD cDNA from pOTB7hMyoD1 (ID 5022419 Open Biosystems) was subcloned as EcoRI/Xhol fragment into pMSCV-ires-GFP to generate pMSCV-hMyoD-ires-GFP.

Retroviral supernatants were produced in 293T cells cultured in DMEM/10\% FBS. Viral constructs were co-transfected with pCL-Eco packaging constructs (Naviaux et al., 1996). Medium was changed after $24 \mathrm{~h}$ and the viral supernatant was collected at $48 \mathrm{~h}$ post-transfection. Filtered supernatant $(0.45 \mu \mathrm{m})$ was applied to the subconfluent cells with polybrene $(4 \mu \mathrm{g} / \mathrm{ml})$. Spin-infection was performed at 2500 r.p.m. at $33{ }^{\circ} \mathrm{C}$ for $90 \mathrm{~min}$. Cells were incubated for additional $12 \mathrm{~h}$ at $37{ }^{\circ} \mathrm{C}$ after which time the supernatant was replaced with fresh medium. Two days post-infection, proliferation medium was switched to myoblast differentiation medium (DMEM with $2 \% \mathrm{HS}$ ) and treatment with doxycycline was started. To evaluate the infection efficiency at day 2 post-transduction, cells were trypsinized and analyzed for GFP expression by flow cytometry (BD FACS Aria). The expression of the inserted gene was also confirmed by RT-PCR.

\section{Statistical analyses}

All experiments were done at least 3 times. Data shown for Real Time RT-PCR and myotubes fusion index are the mean \pm STDEV. Difference between means was compared by the two-tailed Student test (GraphPad Prism 5) and was considered significantly different at $P<0.05$.

\section{Acknowledgments}

We thank the Dr. Bob and Jean Smith Foundation for their generous support. This work was also supported in part by a Muscular Dystrophy Association Fellowship (to D.B.), the Facioscapulohumeral Muscular Dystrophy Society, and a U.T. Southwestern S.U.R.F. Award (to S.L.).

\section{References}

Ansseau, E., Laoudj-Chenvesse, D., Marcowycz, A., Sauvage, S., Barro, M., Tassin, A., Matteotti, C., van Acker, A., Leroy, A., Leclercq, I., Leo, O., Figlewicz, D., Belayew, A., Coppee, F., 2006. Characterization of the DUX4c gene located within a repeated element close to the FSHD locus. Neuromuscul. Disord. 16, 644-726.

Bajard, L, Relaix, F, Lagha, M., Rocancourt, D., Daubas, P, Buckingham, M.E, 2006. A novel genetic hierarchy functions during hypaxial myogenesis: Pax3 directly activates Myf5 in muscle progenitor cells in the limb. Genes Dev. 20, 2450-2464.

Buckingham, M., Bajard, L., Chang, T., Daubas, P., Hadchouel, J., Meilhac, S., Montarras, D., Rocancourt, D., Relaix, F., 2003. The formation of skeletal muscle: from somite to limb. J. Anat. 202, 59-68.

Celegato, B., Capitanio, D., Pescatori, M., Romualdi, C., Pacchioni, B., Cagnin, S., Vigano, A., Colantoni, L., Begum, S., Ricci, E., Wait, R., Lanfranchi, G., Gelfi, C., 2006. Parallel protein and transcript profiles of FSHD patient muscles correlate to the D4Z4 arrangement and reveal a common impairment of slow to fast fibre differentiation and a general deregulation of MyoD-dependent genes. Proteomics 6, 5303-5321.

Dixit, M., Ansseau, E., Tassin, A., Winokur, S., Shi, R., Qian, H., Sauvage, S., Matteotti, C., van Acker, A.M., Leo, O., Figlewicz, D., Barro, M., Laoudj-Chenivesse, D., Belayew, A., Coppee, F., Chen, Y.W., 2007. DUX4, a candidate gene of facioscapulohumeral muscular dystrophy, encodes a transcriptional activator of PITX1. Proc. Natl. Acad. Sci. U. S. A. 104, 18157-18162.

Gabellini, D., Green, M.R., Tupler, R., 2002. Inappropriate gene activation in FSHD: a repressor complex binds a chromosomal repeat deleted in dystrophic muscle. Cell $110,339-348$.

Gabellini, D., D'Antona, G., Moggio, M., Prelle, A., Zecca, C., Adami, R., Angeletti, B., Ciscato, P., Pellegrino, M.A., Bottinelli, R., Green, M.R., Tupler, R., 2006. Facioscapulohumeral muscular dystrophy in mice overexpressing FRG1. Nature 439, 973-977. 
Gabriels, J., Beckers, M.C., Ding, H., De Vriese, A., Plaisance, S., van der Maarel, S.M. Padberg, G.W., Frants, R.R., Hewitt, J.E., Collen, D., Belayew, A., 1999. Nucleotide sequence of the partially deleted D4Z4 locus in a patient with FSHD identifies a putative gene within each $3.3 \mathrm{~kb}$ element. Gene 236, 25-32.

Hessabi, B., Ziegler, P., Schmidt, I., Hessabi, C., Walther, R., 1999. The nuclear localization signal (NLS) of PDX-1 is part of the homeodomain and represents a novel type of NLS. Eur. J. Biochem. 263, 170-177.

Hewitt, J.E., Lyle, R., Clark, L.N., Valleley, E.M., Wright, T.J., Wijmenga, C., van Deutekom, J.C., Francis, F., Sharpe, P.T., Hofker, M., et al., 1994. Analysis of the tandem repeat locus D4Z4 associated with facioscapulohumeral muscular dystrophy. Hum. Mol. Genet. 3, 1287-1295.

Kowaljow, V., Marcowycz, A., Ansseau, E., Conde, C.B., Sauvage, S., Matteotti, C., Arias, C., Corona, E.D., Nunez, N.G., Leo, O., Wattiez, R., Figlewicz, D., Laoudj-Chenivesse, D., Belayew, A., Coppee, F., Rosa, A.L., 2007. The DUX4 gene at the FSHD1A locus encodes a pro-apoptotic protein. Neuromuscul. Disord. 17, 611-623.

Naviaux, R.K., Costanzi, E., Haas, M., Verma, I.M., 1996. The pCL vector system: rapid production of helper-free, high-titer, recombinan retroviruses. J. Virol. 70, 5701-5705.

Ostlund, C., Garcia-Carrasquillo, R.M., Belayew, A., Worman, H.J., 2005. Intracellular trafficking and dynamics of double homeodomain proteins. Biochemistry 44, 2378-2384.

Rudnicki, M.A., Schnegelsberg, P.N., Stead, R.H., Braun, T., Arnold, H.H., Jaenisch, R., 1993. MyoD or Myf-5 is required for the formation of skeletal muscle. Cell 75 1351-1359.
Tajbakhsh, S., Rocancourt, D., Cossu, G., Buckingham, M., 1997. Redefining the genetic hierarchies controlling skeletal myogenesis: Pax-3 and Myf-5 act upstream of MyoD. Cell 89, 127-138.

Weintraub, H., Davis, R., Tapscott, S., Thayer, M., Krause, M., Benezra, R., Blackwell, T.K., Turner, D., Rupp, R., Hollenberg, S., et al., 1991. The Myod gene family: nodal point during specification of the muscle cell lineage. Science 251, 761-766.

Wijmenga, C., Hewitt, J.E., Sandkuijl, L.A., Clark, L.N., Wright, T.J., Dauwerse, H.G., Gruter, A.M., Hofker, M.H., Moerer, P., Williamson, R., et al., 1992. Chromosome 4q DNA rearrangements associated with facioscapulohumeral muscular dystrophy. Nat. Genet. 2, 26-30.

Winokur, S.T., Barrett, K., Martin, J.H., Forrester, J.R., Simon, M., Tawil, R., Chung, S.A Masny, P.S., Figlewicz, D.A., 2003a. Facioscapulohumeral muscular dystrophy (FSHD) myoblasts demonstrate increased susceptibility to oxidative stress. Neuromuscul. Disord. 13, 322-333.

Winokur, S.T., Chen, Y.W., Masny, P.S., Martin, J.H., Ehmsen, J.T., Tapscott, S.J., van der Maarel, S.M., Hayashi, Y., Flanigan, K.M., 2003b. Expression profiling of FSHD muscle supports a defect in specific stages of myogenic differentiation. Hum. Mol. Genet. 12, 2895-2907.

Wright, T.J., Wijmenga, C., Clark, L.N., Frants, R.R., Williamson, R., Hewitt, J.E., 1993. Fine mapping of the FSHD gene region orientates the rearranged fragment detected by the probe p13E-11. Hum. Mol. Genet. 2, 1673-1678. 